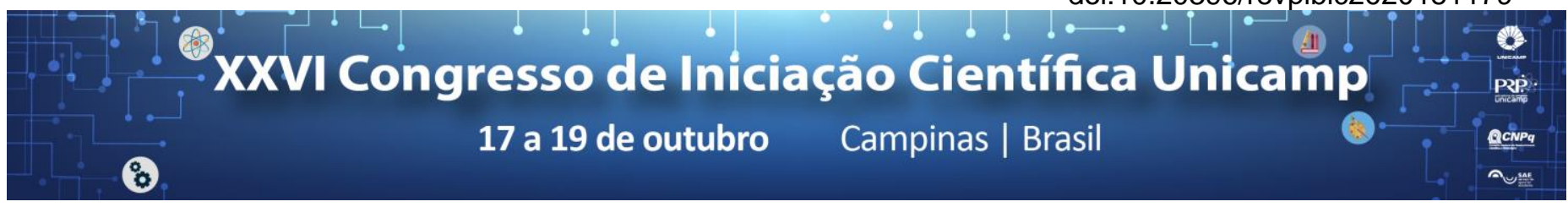

\title{
MICROBIOTA-DERIVED METABOLITES, SHORT CHAIN-FATTY ACIDS, INDUCE EPIGENETIC MODIFICATIONS IN CD4+ T CELLS IN MICE.
}

\author{
Raissa C.A. Silva*, Laís P. Pral, José L. Fachi, Marco A. R. Vinolo
}

\begin{abstract}
The gut microbiota is indispensable for the host, considering its role in regulating key aspects of host homeostasis, such as development, function and induction of $T$ cells. One of the possibilities for microbiota-host interaction is through short-chain fatty acids (SCFAs). compounds produced by fermentation of dietary fiber of intestinal lumen bacteria. These compounds can regulate gene expression, by promoting inhibition of histone deacetylase enzimes (HDACs) and activation of histone acetyltransferases enzimes (HATs), thus increasing post-translational modifications such as acetylation and crotonylation. However, details of how these two types of modifications act on host cells, especially regulatory $T$ cells, remains to be seen. Therefore, our aim was to evaluate the SCFA mediated role of microbiota on acetylation and crotonylation of regulatory $T$ cells, as to verify how these two modifications can interact in the histone modification scenario. In conclusion, acetylation and crotonylation, linked to microbiota, have the potential to form an importante part in regulation of T CD4+ cells and can interact and modify directly the action of HDAC enzimes, which is notably relevant to microbiota-host interface.
\end{abstract}

\section{Key words: \\ Microbiota; short-chain fatty acids; $T$ cells.}

\section{Introduction}

The microbial communities from intestinal tract include bacteria, virus, Archaea and fungi, collectively known as microbiota. ${ }^{1}$ The gut microbiota is indispensable for the host, considering its role in regulating key aspects of host homeostasis, such as metabolism, cell development and gene expression, besides shaping function of intestinal epithelial cells, mucosal barrier components ${ }^{2}$ and the development, function and induction of $\mathrm{T}_{\text {cells }}^{3}$ Still, the host defense system is essential for controlling gut colonization by microbiota elements ${ }^{1}$. One of the possibilities for microbiota-host interaction is through short-chain fatty acids (SCFAs), compounds produced by fermentation of dietary fiber of intestinal lumen bacteria. The main SCFAs found in human gut are acetate, propionate and butyrate, all of them the deprotonated form, due to physiological $\mathrm{pH}$, of their respective acids, which are the actual bacteria metabolite ${ }^{4}$. These compounds can regulate gene expression, by promoting inhibition of histone deacetylase enzymes (HDACs) and activation of histone acetyltransferases enzymes (HATs), thus increasing post-translational modifications such as acetylation and crotonylation. Besides direct regulation, there is evidence to support that some modifications can occur in a non enzymatic manner, as a result from direct contact between metabolized SCFAs and proteins. It is important to indicate that the alterations acetate, crotonate and others SCFAs induce on histone and nonhistone proteins are reversible and very relevant, for its effects on gene expression constitute a fast modulation mechanism for several genes ${ }^{5,6}$. However, details of how these two types of modifications act on host cells, especially regulatory $\mathrm{T}$ cells, and how they interact with one another to modify histones, remains unknow. Therefore, our aim was to evaluate the SCFA mediated role of microbiota on acetylation and crotonylation of regulatory $T$ cells, as to verify how these two modifications can interact in the histone modification scenario.

\section{Results and Discussion}

We harvested spleen T CD4+ cells from 8 weeks old C57BL/ 6 mice for 6 hours under $37^{\circ} \mathrm{C}$ with $2 \mathrm{mM}$ of vorinostat, a HDAC inhibitor, and $20 \mathrm{mM}$ of crotonate. Total protein analysis by Western Blotting showed that, in comparison with control, individual treatments with crotonate and HDAC inhibitor increased crotonylation and acetylation in CD4+ cells, from which the inhibitor presented stronger enhancer effects. The presence of both treatments together also increased crotonylation and acetylation to a lesser extent, if compared to the individual treatments.

\section{Conclusions}

In conclusion, acetylation and crotonylation, linked to microbiota, have the potential to form an importante part in regulation of $\mathrm{T}$ CD4+ cells and can interact and modify directly the action of HDAC enzimes, which is notably relevant to microbiota-host interface.

\footnotetext{
${ }^{1}$ Kaiko GE, Stappenbeck TS. Host-microbe interactions shaping the gastrointestinal environment. Trends in immunology. 2014, 35(11): 538-548.

${ }^{2}$ Hooper L. V., Macpherson A. J. Immune adaptations that maintain homeostasis with the intestinal microbiota. Nat. Rev. Immunol. 2010, 10:159-169.

3 Lee N, Kim W-U. Microbiota in T-cell homeostasis and inflammatory diseases. Experimental \& Molecular Medicine. 2017, 49(5)

${ }^{4}$ Corrêa-Oliveira, Renan et al. Regulation of Immune Cell Function by ShortChain Fatty Acids. Clinical \& Translational Immunology 5.4. 2016, e73-

${ }^{5}$ Kaiko G.E. et al. The Colonic Crypt Protects Stem Cells from Microbiota-Derived Metabolites. Cell. 2016, 165(7): 1708-1720.

${ }^{6}$ Donohue, DR et al. The Warburg Effect Dictates the Mechanism of Butyrate Mediated Histone Acetylation and Cell Proliferation. Mol Cell. 2012, 48(4): $612-$ 626.
} 